\title{
Evaluation of direct immunofluorescent method in the detection of Pneumocystis jiroveci among patients with lower respiratory tract infections
}

\author{
Manahil M. Yehia, Asmaa Z. Sheetawy Zainalabideen A. Abdulla \\ Department of Microbiology, College of Medicine, University of Mosul, Mosul, Iraq \\ Correspondence: dr.manahil2012@yahoo.com

$\frac{\text { Received }}{24.10 .2013} \quad \frac{\text { Accepted }}{22.1 .2014}$

\begin{abstract}
Objective: Evaluate the use of direct immunofluorescent method in the detection of Pneumocystis jiroveci from induced sputum of patients with LRT infections.

Patients and methods: Thirty patients with LRT infections were included (24 patients were immunocompromised and 6 immunocompetent). The present study conducted for 6 months from January-June, 2011. Smears were prepared from their induced sputa and stained with direct fluorescent antibody stain, then examined under fluorescent microscope.

Results: Sputum of 5(16.7\%) patients revealed positive results for the presence of $P$. jiroveci and all of them were immunocompromised (two acute myloid leukemia, one acute lymphoid leukemia, one non-Hodgkin's lymphoma, and one asthma under long coarse of corticosteroid therapy).
\end{abstract}

Conclusion: Direct fluorescent antibody technique is more sensitive than the conventional methods for the detection of P. jiroveci in LRT infections.

Keywords: Pneumocystis pneumoniae, Pneumocystis jiroveci, immunocompromised patients.

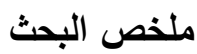

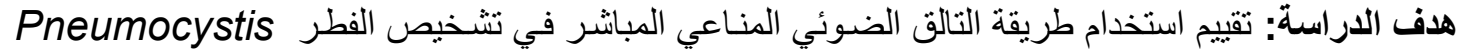

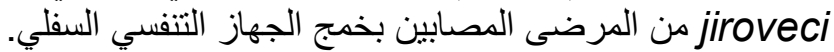
المرضى وطرائق العمل: تضمنت هذه الدراسة 30 مريضا يعانون من خمج الجهاز الجهاز التنفسي السفلي،24

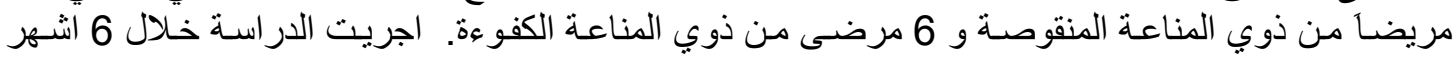

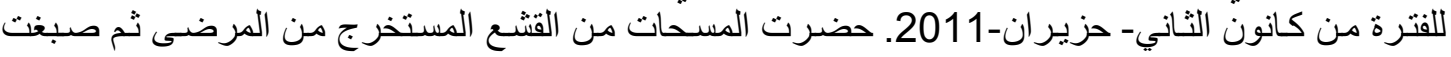

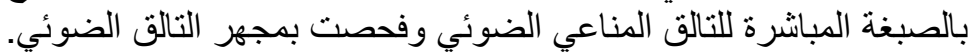

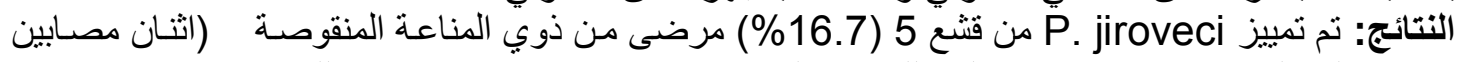

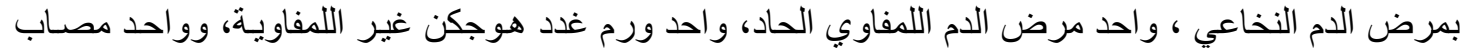

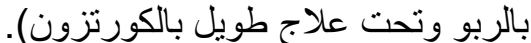
P. الاستنتاج: ان طريقة التالق الضـوئي المناعي المباشتر حساسـة اكثر من الطرق التقليديـة لاستكثـاف فطر jiroveci 
$\mathbf{P}$ neumocystis jiroveci (formly carinii), remains an important cause of pneumonia ${ }^{1}$. Immunocompromised patients with HIV infection, hematologic malignancies, prolonged corticosteroid therapy, or intensive immunosuppressive therapy are under risk of infection with P. jiroveci ${ }^{2,3}$.

Microscopic identification of the organism be achieved from clinical samples of sputum, bronchoalveolar large (BAL), or lung biopsy ${ }^{4}$. The conventional methods for the detection of P. jiroveci is generally established by morphologically demonstrating pneumocystis organism by different methods. These methods were Giemsa or Gram-Weigert stain for trophozoites and Gomori methenamine silver stain, toludine blue or calcofluor white for cysts $^{5,6}$. A more sensitive and rapid diagnostic method is the imunofluorescent stains (direct and indirect fluorescent antibody). The direct fluorescent antibody (DFA) has a higher sensitivity and specificity for the detection of pneumocystis ${ }^{7}$, while the indirect antibody technique gives a wider range of detection ${ }^{8}$. Furthermore, PCR-based amplification assays has an increased sensitivity for the detection of P. jiroveci compared to other tests ${ }^{9}$.

The main aim of the present study is to evaluate the use of DFA technique in the detection of $P$. jiroveci from induced sputum of the patients with lower respiratory tract (LRT) infections.

\section{Patients and Methods}

Thirty patients with LRT infections were included. Twenty four patients were presumptively immunocompromised (with underlying diseases) and 6 were immunocomptent (without underlying diseases). The present study conducted for 6 months from January-June, 2011. The studied patients were hospitalized in Ibn-Sina
Teaching Hospital, and Oncology and Nuclear Medicine Hospital, Mosul Iraq. The immunocompromised patients had different underlying diseases including various types of leukemia ${ }^{11}$, non Hodgkin's lymphoma ${ }^{6}$ multiple myloma ${ }^{2}$, bicyclic neutropenia ${ }^{2}$, bronchogenic carcinoma $^{1}$, and asthmatic patients with prolonged corticosteroid therapy ${ }^{2}$. Patients with malignancies were under chemo and / or radiation therapy. The duration of the underlying causes of immunosuppression in these patients ranged from 6 months-16 years. However, the duration of LRT infection at the time of sampling of the patients were ranged from 5 days to 3 months. Most patients have low grade fever, dry cough with minimal frothy sputum and shortness of breath.

\section{Sampling and preparation of the specimens}

The induced sputa were collected at the first morning in steril containers after induction by hypertonic saline $(3 \%)$ and sent to the laboratory within 1 hour. The samples were shaked by vortex for 1-2 minutes for homogenization. Smears were prepared and fixed in acetone for 10 minutes. The DFA staining was performed, using a commercial kit for the detection of $P$. jiroveci purchased from Meri-Fluor- pneumocystis catalog No. 222030, Meridian, Bioscience, Inc, Ohio USA. Fluorescein isothiocyanate (FITC) labeled monoclonal antibody (McA) was applied for the detection of P. jiroveci (cysts, sporozoites, and trophozoites), and the staining procedure was conducted according to the manufacturer's instructions.

\section{Results}

The age of the 30 studied patients ranged from 18-71 (mean $\pm \mathrm{SD}=$ $42.03 \pm 17.1)$ years. The males were 11 and females were 19. 
Sputum of $5(16.7 \%)$ out of 30 patients with LRT infections showed positive results for $\mathrm{P}$. jiroveci, and all of them were immunocompromised. Under high power magnification of the fluorescent microscope, either the trophozoites, cysts or both were detected in the positive samples (Fig. I).
The clinical data of the 5 patients with positive results for $P$. jiroveci are summarized in Table I. The patients were 3 females, and 2 males, and their ages ranged between 35-71 years. Two of the patients were with acute myloid leukemia, one with acute lymphoid leukemia, one with non-Hodgkin's lymphoma, and one with asthma under long coarse of corticosteroid therapy.

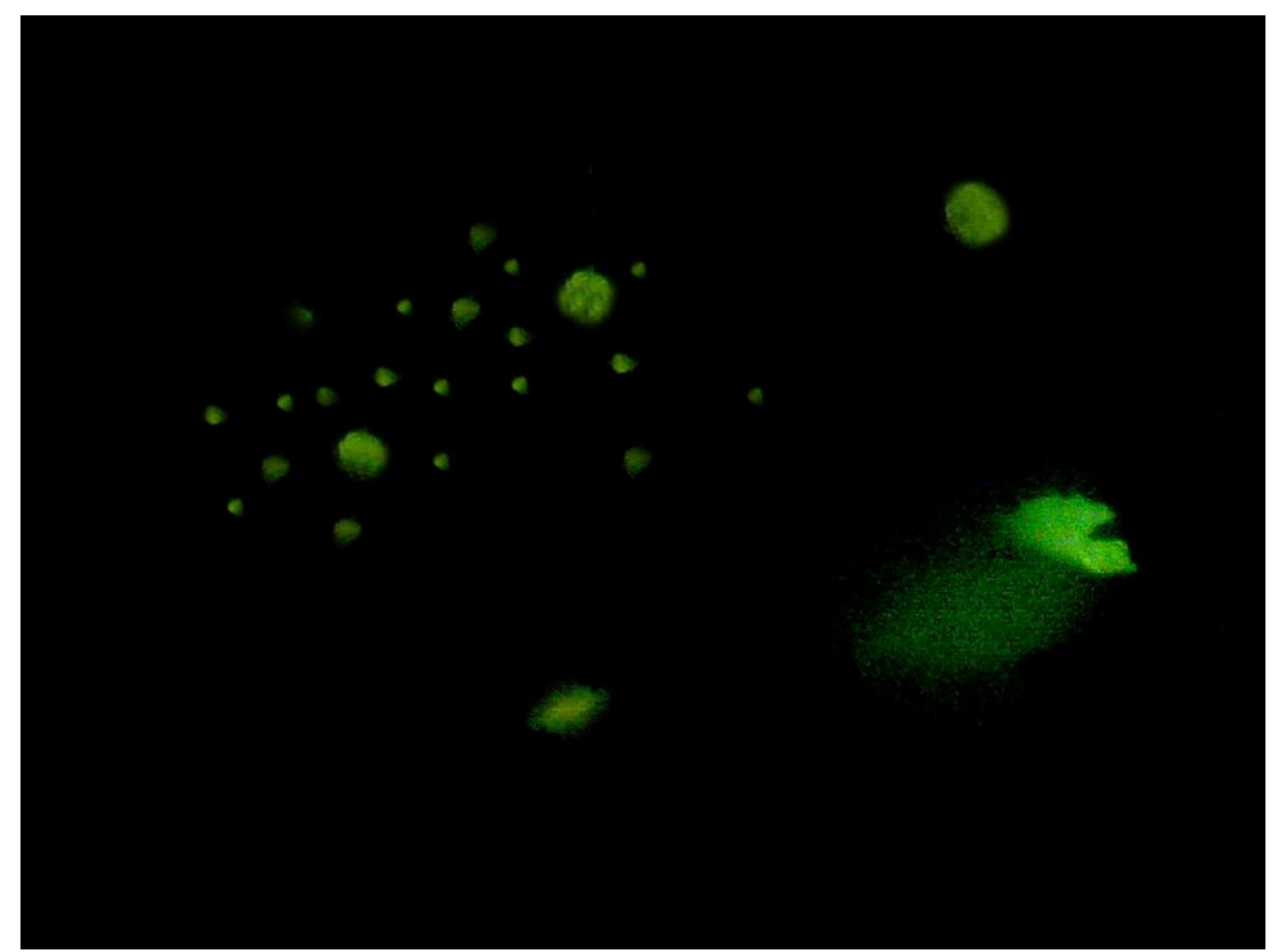

Figure 1. Pneumocystis jiroveci (cysts, trophozoites) in induced sputum stained with direct fluorescent antibody stain, under fluorescent microscope (40X). 
Table 1. Clinical data of patients with positive results for $P$. jiroveci

\begin{tabular}{|l|c|}
\hline \multicolumn{1}{|c|}{ Items } & No. \\
\hline 1. No. of cases & 5 \\
\hline $\begin{array}{l}\text { 2. Gender } \\
\text { males } \\
\text { females }\end{array}$ & 2 \\
\hline 3. Age & 3 \\
\hline 4. Immunosuppressive diseases & $35-71$ \\
- acute myloid leukemia. & 2 \\
- acute lymphoid leukemia. & 1 \\
- non-Hodgkin's lymphoma & 1 \\
- asthma + corticosteroid & 1 \\
\hline 5. Immunosuppressive therapy & 4 \\
- chemotherapy & 1 \\
\hline$\quad$ corticosteroid & 6 month-16 Years \\
\hline 6. Duration of the disease & 4 \\
\hline 7. Symptom and signs & 4 \\
\hline$\quad$ fever & \\
• dry cough & 4 \\
\hline 8. Croase appearance of the sputum & 1 \\
\hline - forthy white & \\
\hline
\end{tabular}

\section{Discussion}

Pneumocystis jiroveci is emerging as an important pathogen in immunocompromised patients ${ }^{10}$. The disease probably occurs either by new acquisition of infection or reactivation of latent infection. Moreover, case clustering among immuosuppressed patients suggest that pneumocystis spread is usually by air borne route ${ }^{11}$. Five immunocompromised individuals out of 30 patients studied revealed the presence of pneumocystis in their seputa when examined by the DFA technique. Fluorescent antibody staining technique is now frequently used for this purpose with high efficiency $^{12}$. The 5 patients positive for P. jiroveci were presumptively immunocompromised for reasons other than HIV (Table I). These results were in keeping with the finding of
Saksasithon and co workers (2003) who reported that the patients who were immunocompromised for reasons such as haemtological malignancies or immunosuppressive therapy for autoimmune disease are also at risk for pneumocystis pneumonia (PCP). Furthermore, the predominant presenting symptoms of the P. jiroveci positive patients were dry cough $(4 / 5)$ and fever (4/5). Other investigators reported that fever was the main presenting symptom in $\mathrm{PCP}^{12}$.

The first report from Mosul -Iraq in 2009 about the identification of $p$. carinii in LRT infections was based on direct identification using Giemsa, toludin blue and calcofluor staining ${ }^{13}$. The present study (from the same geographical area) applied IF staining using monoclonal antibody for the identification of P. jiroveci. There was a higher frequency of $P$. jiroveci 
identification between the first and the current report $(8.6 \%$ Vs $16.7 \%$ respectively). Flood and coworkers (1991) mentioned that DFA was more sensitive than the conventional method (48\% Vs 39\%) in the detection of P. jiroveci. Other workers reported that the frequency of $\mathrm{P}$. jiroveci detection in clinical respiratory specimens by Merifluor pneumocystic stain was $90.8 \%{ }^{15}$. The DFA has also the advantage of being less time consuming, although more expensive than the conventional methods.

In conclusion, the method of DFA technique has a superior diagnostic utility than the conventional methods for the detection of $P$. jiroveci among immunocompromised patients with LRT infections.

\section{References}

1. Saksasithon S, Sungkanuparph S, Thanakitcharu S. Pneumocystis jiroveci pneumonia in patients without HIV infection. J Med Assoc Thai 2003; 86: 612-616.

2. De Bock R, Middelheim AZ. Febrile neutropenia in allogeneic transplantataion. Int $\mathrm{J}$ Antimicrob Agents 2000; 16:177-180.

3. Rolston KVI. The spectrum of pulmonary infections in cancer patients. Curr opin oncol 2001;13:218223.

4. Jones CIA. Pneumocystis pneumonia. Gale encyclopedia of medicine. Published by Gale group, Amer Lung Assoc 2002; 800: 586-872.

5. Thomas CF, Limper AH. Pneumocystis pneumonia. New Eng J Med 2004; 350(24):2487-2498.

6. Caliendo AM, Hewitt PL, Allega JM et al. Performance of a PCR assay for detection of Pneumocystis carinii from respiratory specimens. J Clin Micro 1998; 36(4):979-982.
7. NG VL, Virani NA, Chaisson RE et al. Rapid detection of pneumocystis carinii using a direct fluorescent monoclonal antibody stain. J Clin Microbiol 1990; 28: 2228-2233.

8. Kirsck CM, Azzi RL, Yenokida GG et al. Analysis of induced sputum in the diagnosis of pneumocystis carinii pneumonia. Am J Med Soci 1999; 386391.

9. Huggett JF, Taylor MS, Kocjan G et al. Development and evaluation of a real-time PCR assay for detection of pneumocystis carinii DNA in bronchoalveolar lavange fluid of HIV -infected patients. Thorax 2008;63: 154-159.

10. Mathews MS and Mathai. E. Emerging importance of Pneumocystis carinii among Indian immunosuppressed patients. Indian J Chest Dise and Allied Sci 2000; 42 (4): 323-324.

11. Keely SP, Stringer JR, Baughman RP., et al. Genetic variation among Pneumocystis carinii hominis isolates in recurrent pneumocystosis. J Infect Dis $1995 ; 172: 595-598$.

12. Koziel H. Pneumocystis carinii, Fungal pathogenesis principles and clinical application. In calderone RA and Cihalar RL, editors. Marcel Dekker, Inc. New York 2002; p 227260.

13. Yehia MM. Identification of fungi in lower respiratory tract infection among immunocompotent and immunocompromised patients, Ph.D Thesis. Mosul University. 2009;138p.

14. Flood D, Rawji M, Gobin G, et al. Direct fluorescent antibodies Vs GMS stain for the detection of Pneumocystis carinii in bronchoalveolar lavage. Int Conf AIDS 1991; June, 7:240.

15. Procop GW, Haddad S, Quinn J, et al. detection of Pneumocystis jiroveci in respiratory specimens by four staining methods. J Clin Microbiol 2004; 42(7) July: 3333-3335. 